Revue musicale OICRM

\title{
Susciter un vaste forum de discussions. Les écrits de Wagner dans la presse allemande des années 1850
}

\section{Jean-François Candoni}

Volume 7, numéro 1, 2020

Écrits de compositeurs et espaces médiatiques

URI : https://id.erudit.org/iderudit/1069469ar

DOI : https://doi.org/10.7202/1069469ar

Aller au sommaire du numéro

\section{Éditeur(s)}

Observatoire interdisciplinaire de création et recherche en musique (OICRM)

\section{ISSN}

2368-7061 (numérique)

Découvrir la revue

Citer cet article

Candoni, J.-F. (2020). Susciter un vaste forum de discussions. Les écrits de Wagner dans la presse allemande des années 1850. Revue musicale OICRM, 7(1), 19-35. https://doi.org/10.7202/1069469ar
Résumé de l'article

Cet article examine comment un espace médiatique s'est constitué autour des écrits de Richard Wagner dans l'Allemagne du milieu du $\operatorname{XIX}^{\mathrm{e}}$ siècle. La contribution prend en compte la réception par la presse allemande de l'ensemble des textes théoriques du compositeur, depuis ses premiers essais jusqu'aux écrits de Zurich consacrés à la réforme de l'opéra. Il apparaît clairement que la réception de ses écrits dans la presse spécialisée et quotidienne et la vivacité des débats qu'ils ont fait naître ont joué un rôle décisif non seulement dans la naissance de phénomène Wagner, mais aussi dans la façon dont son oeuvre dramatique a été perçue à travers des analyses parfois précises, mais souvent réductrices, voire erronées. 


\title{
Susciter un vaste forum de discussions. Les écrits de Wagner dans la presse allemande des années 1850
}

\author{
Jean-François Candoni
}

\begin{abstract}
Résumé
Cet article examine comment un espace médiatique s'est constitué autour des écrits de Richard Wagner dans l'Allemagne du milieu du XIX siècle. La contribution prend en compte la réception par la presse allemande de l'ensemble des textes théoriques du compositeur, depuis ses premiers essais jusqu'aux écrits de Zurich consacrés à la réforme de l'opéra. Il apparaît clairement que la réception de ses écrits dans la presse spécialisée et quotidienne et la vivacité des débats qu'ils ont fait naître ont joué un rôle décisif non seulement dans la naissance de phénomène Wagner, mais aussi dans la façon dont son œuvre dramatique a été perçue à travers des analyses parfois précises, mais souvent réductrices, voire erronées.
\end{abstract}

Mots clés : antisémitisme ; critique musicale ; presse ; réforme de l'opéra ; Richard Wagner.

\begin{abstract}
This article examines how a media space was built around Richard Wagner's writings in mid-19th century Germany. The contribution takes into account the reception by the German press of all the composer's theoretical texts, from his first essays to Zurich's writings on opera reform. It is clear that the reception of his writings in the specialized and daily press and the liveliness of the debates they gave rise to play a decisive role not only in the birth of the Wagner phenomenon, but also in the way in which his dramatic work was perceived through analyses that were sometimes precise, but often reductive or even erroneous.

Keywords : antisemitism; music criticism; press; reform of the opera; Richard Wagner.
\end{abstract}


Richard Wagner a très tôt essayé de constituer autour de lui une communauté de partisans. Afin de rallier des adeptes à ses projets de réforme de l'opéra, il s'est servi des moyens de communication et de publicité les plus modernes de son époque. C'est dans cette optique qu'il s'est lancé dans la rédaction de ses innombrables essais, écrits théoriques et critiques musicales consacrés aux sujets les plus divers. Ces textes - ou du moins certains d'entre eux - ont eu un large écho auprès de ses contemporains. On peut même affirmer que l'incroyable notoriété qu'a connue le compositeur de Lohengrin à partir de la seconde moitié du XIX ${ }^{\mathrm{e}}$ siècle ne s'explique pas uniquement par le caractère de nouveauté de ses drames musicaux ou par le soutien décisif que lui ont apporté d'influents mécènes (Pauline von Metternich, le roi Louis II) et des artistes de renom (Liszt, Baudelaire), mais également par la résonance médiatique de ses essais en tout genre. Si Wagner a suscité l'intérêt d'un assez large public, c'est également parce que ses écrits - dont ses opéras seraient l'émanation - ne parlent pas seulement de musique et de théâtre, mais abordent en même temps un certain nombre des problématiques sociales, politiques et existentielles qui cristallisent les préoccupations de l'époque et proposent in fine une manière d'utopie artistique et sociale qui - peut-être à cause de son caractère nébuleux (et parfois franchement douteux) - a fasciné ses contemporains autant qu'elle les a irrités.

Wagner a su habilement instrumentaliser la presse - aussi bien musicale que généraliste -, qui a connu dans l'Allemagne de la seconde moitié du XIX ${ }^{\mathrm{e}}$ siècle un essor considérable malgré la pression permanente de la censure, même si cette dernière se desserre légèrement après la révolution de 1848 (Nipperdey 2013, t. 1, p. 592). Il y publie un nombre important d'essais et d'articles. On constate même que, jusqu'aux « écrits de Zurich » du début des années 1850, la quasi-totalité de ses textes, y compris son Esquisse autobiographique ${ }^{1}$ de 1843, sont parus dans des périodiques. Il est évident que - bien qu'il ait prétendu le contraire avec une certaine véhémence ${ }^{2}$ - la presse a offert à ses opéras et à ses écrits une publicité considérable. Au cours des années qui voient Richard Wagner devenir un important phénomène médiatique c'est-à-dire entre 1842 (année de la création de Rienzi) et 1852 (année de la parution des articles de Theodor Uhlig sur les écrits théoriques du compositeur) -, la presse

\footnotetext{
1 Intitulé "Autobiographische Skizze » dans les Gesammelte Schriften und Dichtungen, le texte est publié initialement dans la Zeitung für die elegante Welt sous le titre " Richard Wagner " (1 $1^{\text {er }}$ février 1843 , p. 114-119; 8 février 1843, p. 135-139).

2 On se reportera, pour se faire une idée plus précise de l'inimitié de Wagner envers les acteurs de la presse, aux textes suivants : " De la critique musicale » ("Über musikalische Kritik », 1852), Sur l'État et la religion (Über Staat und Religion, 1864), Éclaircissements sur le Judaïsme dans la musique (Aufklärungen über das Judenthum in der Musik, 1869), Le carnet brun. Journal (Das braune Buch. Tagebuchaufzeichnungen, 1865-1882). Dans le texte de 1864, sa mauvaise foi atteint son paroxysme lorsqu'il écrit : " Il n'est peut-être pas aussi paradoxal qu'il y paraît d'affirmer qu'avec l'invention de l'imprimerie et, plus certainement encore, avec le développement du journalisme, l'humanité a imperceptiblement perdu de sa capacité à émettre des jugements sains " (Sämtliche Schriften und Dichtungen $[=\underline{S S D}]$, t. 8, p. 17 : "Es ist gewiß nicht so paradox, als es den Anschein hat, zu behaupten, daß mit der Erfindung der Buchdruckerkunst, ganz gewiß aber mit dem Aufkommen des Zeitungswesens, die Menschheit unmerklich von ihrer Befähigung zu gesundem Urtheile verloren hat »). La traduction de l'ensemble des textes en allemand a été réalisée par nos soins.
} 
germanophone publie plus de 2000 articles ayant pour sujet Richard Wagner ${ }^{3}$. Cela va de l'article d'une dizaine de lignes à l'essai de 50 pages - et ce chiffre ne tient pas compte des nombreux encarts publicitaires destinés à faire connaître telle ou telle publication ou composition du maître ${ }^{4}$.

Le rapport de Wagner à la presse était toutefois très ambigu, plein de malentendus, et le regard qu'il portait sur les journalistes était extrêmement critique. Il ressent chez ces derniers une « hostilité manifeste se traduisant par une dépréciation de chacune de [s] es productions artistiques, en particulier dans la presse quotidienne, pas seulement en Allemagne, mais également en France et en Angleterre ${ }^{5}$ " (Wagner 1912b, p. 239). Son extrême méfiance envers les journalistes et critiques musicaux s'exprime notamment dans un article en forme d'essai intitulé « De la critique musicale. Lettre au rédacteur en chef de la Neue Zeitschrift für Musik ${ }^{6}$ "(Wagner 1912a, p. 59) dans lequel il explique, de manière assez spécieuse, que la critique n'a selon lui de légitimité qu'à deux conditions : dans un premier temps, elle doit dénoncer une situation dans laquelle la musique a besoin de la critique pour être comprise ; et dans un second temps, la critique doit permettre à la musique d'atteindre le stade " où elle n'aura plus besoin de la médiation de la littérature critique pour être comprise ${ }^{7}$ " (ibid.). Mais si Wagner méprisait - ou plutôt : faisait mine de mépriser - la presse, elle lui a indiscutablement été d'une grande utilité et lui a malgré tout permis d'arriver à ses fins - c'est-à-dire attirer l'attention du public et des intellectuels sur son œuvre et nourrir le débat autour de ses positions esthétiques et idéologiques -, fût-ce d'une façon peu orthodoxe. Il utilise en effet la presse d'une manière très différente de son contemporain Giacomo Meyerbeer, qu'il accusait de soudoyer les journalistes ; ce dernier déployait effectivement d'importants efforts pour établir de bonnes relations avec les critiques et les artistes influents et s'assurer sinon de leur soutien, du moins de leur écoute (Zimmermann 1998, p. 181-195 ; Döhring 2014, p. 38). De son côté, l'auteur de Lohengrin ne faisait pas véritablement de lobbying, mais il avait adopté, inconsciemment peut-être, une stratégie foncièrement différente : il misait sur le

\footnotetext{
3 Pour un premier aperçu de la réception des opéras de Wagner dans la presse allemande dans les années 1840 et 1850, voir Bermbach 2013, p. 162-176.

4 Les articles consacrés à Wagner dans la presse allemande entre 1842 et 1852 ont été recensés dans : Kirchmeyer 1967-1985, t. 2-5. La publication de cette importante compilation d'articles de presse a été interrompue faute de moyens financiers. Le chiffre donné par Kirchmeyer est assurément sous-évalué, puisque, d'une part, certaines publications ont été omises dans ce recensement (notamment la fameuse Illustrirte Zeitung) et que, d'autre part, une partie importante des archives de la presse quotidienne a disparu au fil des aléas de l'histoire.

$5 \quad$ "Vor Kurzem wurde mir [...] Ihre verwunderungsvolle Frage nach dem Grunde der Ihnen unbegreiflich dünkenden, so ersichtlich auf Herabsetzung ausgehenden Feindseligkeit berichtet, welcher jede meiner künstlerischen Leistungen namentlich in der Tagespresse, nicht nur Deutschlands, sondern auch Frankreichs und selbst Englands, begegne».

6 «Über musikalische Kritik, Brief an den Herausgeber der „Neuen Zeitschrift für Musik“ ». Le texte est paru initialement sous le titre « Ein Brief an den Redacteur der Neuen Zeitschrift für Musik» dans la Neue Zeitschrift für Musik [= NZfM], 6 février 1852, p. 57-63.

7 "[Die Stellung] in welcher sie dereinst der literarischen Vermittelung zu ihrem Verständnisse eben nicht mehr bedürfen soll».
} 
radicalisme et le caractère provocateur de ses idées pour faire parler de lui, étant manifestement convaincu que toute publicité, même si elle n'était pas laudative, allait accroître sa notoriété, et lui permettrait in fine d'imposer ses vues.

Et c'est en ce sens que ses écrits théoriques ont joué un rôle déterminant. Peu importe, dès lors, que leur contenu corresponde totalement ou partiellement à des convictions artistiques et politiques profondes, le fait est que leur caractère résolument provocateur (que ce soit dans le ton employé ou dans les propositions qui y sont formulées) a été fructueux du point de vue de la stratégie communicationnelle ${ }^{8}$ : les débats qu'ils ont suscités et l'espace médiatique qu'ils ont ouvert ont largement contribué à établir sa renommée. Et cet espace médiatique ne se résume pas à un cercle restreint d'intellectuels et de musicologues. Ses déclarations sur la caducité du genre de l'opéra et sur l'inanité du Grand Opéra français ont été largement relayées par la presse, y compris quotidienne (où son discours a souvent été outrageusement schématisé, voire caricaturé), et l'ont placé au cœur de toutes les discussions sur le théâtre musical.

Il serait faux en effet de croire que la diffusion des écrits de Wagner s'est faite exclusivement par le biais de la presse spécialisée - même si celle-ci est particulièrement prospère au milieu du XIX ${ }^{\mathrm{e}}$ siècle - et à l'attention d'une élite musicale et intellectuelle. Certes, les premières recensions importantes rédigées avec l'intention de faire la réclame de la réforme wagnérienne sont publiées dans la fameuse Neue Zeitschrift für Musik, qui avait clairement choisi, sous l'égide de Franz Brendel, de devenir l'organe officiel de la propagande wagnérienne ${ }^{9}$ : on pense ici aux essais de Uhlig, à ceux de Richard Pohl et à ceux de Brendel lui-même. (On mettra toutefois de côté les textes de Franz Liszt, qui ont évidemment joué un rôle décisif dans la diffusion de l'esthétique de Wagner, mais qui sont pour l'essentiel centrés sur ses opéras, et ne s'intéressent guère à ses essais.) Outre cette revue, certes prestigieuse, mais dont le lectorat était numériquement réduit, c'est surtout la presse généraliste qui s'intéresse aux essais de Wagner et en publie des comptes rendus détaillés à l'occasion de leur publication, leur assurant ainsi une large publicité. La fameuse Illustrirte Zeitung de Leipzig consacre par exemple régulièrement de longs articles aux opéras de Wagner et à ses écrits.

Il est par ailleurs délicat, si l'on veut aborder la question de l'espace médiatique d'un point de vue géographique, de chercher à identifier des centres musicaux qui seraient plutôt hostiles, ou au contraire plutôt favorables à Wagner : on trouve aussi bien dans la presse berlinoise que dans la presse saxonne (Leipzig étant la capitale de l'édition et de la presse au sein de la Confédération germanique) des avis assez divergents. On peut néanmoins affirmer que, même en mettant de côté les critiques

$8 \quad$ Il n'est évidemment pas question de minimiser l'importance des écrits de Wagner dans les débats esthétiques et idéologiques de l'époque. Cet aspect ne pouvant être traité dans le cadre de la présente étude centrée sur les stratégies communicationnelles développées par le compositeur, on pourra se reporter aux différents articles rédigés par nos soins sur les principaux essais du compositeur dans Picard 2010.

9 Dans son éditorial du début de l'année 1852 (NZfM, 2 janvier 1852, p. 1-4), Franz Brendel déclare que la revue a vocation à soutenir le projet de Wagner. Il ne fait ainsi qu'officialiser une tendance déjà à l'œuvre depuis plusieurs années. 
d'Eduard Hanslick, unanimement considéré comme le plus farouche adversaire du compositeur, la presse viennoise lui est résolument hostile, comme en témoigne de manière presque caricaturale un article de la Wiener Zeitung du 30 avril 1850, intitulé "Richard Wagner. L'Euvre d'art de l'avenir » (Anonyme 1850b), dans lequel le compositeur est vilipendé pour ses idées politiques et philosophiques. Il est de manière générale assez difficile de trouver dans la presse viennoise des années 1850 un article donnant une image positive des essais du compositeur, qualifiés par la Wiener Zeitung $\mathrm{d}^{\prime}$ " apothéose du non-sens ${ }^{10}$ " (ibid., [p. 1]). L'avis émis par la Deutsche Zeitung de Vienne lors de la création locale de Tristan et Isolde résume bien, quant à lui, la tonalité générale de la presse autrichienne : "Wagner est une victime de sa théorie et de ses implications ${ }^{11}$ » (Bagge 1861, p. 67).

Étant donné l'ampleur des commentaires suscités par les écrits théoriques de Wagner (il n'est pas question ici de sa production littéraire, constituée pour l'essentiel de livrets d'opéras), nous avons choisi, tout en tenant compte de la première période de l'activité de Wagner (1834-1848), de centrer notre enquête sur les années 1850, c'est-à-dire sur l'époque qui suit immédiatement la parution des " écrits de Zurich ", rédigés à la suite de l'échec de la révolution de 1848/1849, à l'époque de l'exil en Suisse. Les principaux "écrits de Zurich ", consacrés à la réforme de l'opéra, sont L'art et la révolution (Die Kunst und die Revolution, 1849), L'ouvre d'art de l'avenir (Das Kunstwerk der Zukunft, 1849) et Opéra et drame (Oper und Drama, 1851). Ce sont d'ailleurs les textes les plus largement commentés dans la presse allemande des années $1850^{12}$, même si l'on trouve également quelques rares comptes rendus de ses essais relatifs à la réforme des théâtres en Saxe (voir Uhlig 1851), ainsi que quelques réactions au pamphlet sur "Le judaïsme dans la musique " (" Das Judenthum in der Musik "). Ce sont les " écrits de Zurich » qui, beaucoup plus que les essais des années 1870, rédigés après le tournant schopenhauerien, ont durablement marqué la réception du phénomène Wagner.

\section{RÉCEPTION TARDIVE DES PREMIERS ÉCRITS (1834-1848)}

Les tout premiers écrits de Wagner, rédigés avant la révolution de $1848^{13}$, n'ont pas vraiment eu de résonance médiatique en Allemagne, et les quelques recensions qui leur ont été consacrées sont relativement tardives (il serait en revanche intéressant d'étudier l'écho qu'ont pu avoir en France les textes publiés dans la Revue et Gazette musicale de Maurice Schlesinger entre 1840 et 1842). Il faut dire que ces premiers textes, qui sont d'un grand intérêt pour comprendre le parcours du compositeur, ne possèdent pas la force novatrice et provocatrice des écrits ultérieurs. Ils ne

10 "Seine Apotheose des Unsinns".

11 "Er [ist] ein Opfer der Theorie und ihrer Consequenzen geworden ».

12 Ce sont même, à l'exception de la recension d'Alfred Kalischer évoquée infra, les seuls textes ayant fait l'objet de commentaires détaillés (plus d'une page) - du moins dans l'espace germanophone dont il est question ici.

13 Pour une liste complète de ces écrits établie par nos soins, voir Picard 2010, p. 2341-2350. 
suscitent un début d'intérêt qu'à partir de la publication de ses Écrits et poèmes réunis (Gesammelte Schriften und Dichtungen, 1871-1883) au début des années 1870. La Neue Berliner Musikzeitung publie alors, en dix livraisons (à chaque fois en première page), une longue discussion intitulée "Dialogue critique sur les Écrits et poèmes réunis de Richard Wagner » (Kalischer 1872a). Rédigé par le rédacteur en chef de la revue, Alfred Kalischer, le texte fait intervenir, dans sa première partie, quatre personnages, parmi lesquels on trouve trois adeptes inconditionnels du maître (Planastes, Kophander et leur Famulus), qui débattent avec un contradicteur, lequel développe un discours très critique sur Wagner (Glaukopides). Le personnage du philosophe Glaukopides, qui va progressivement dessiller les yeux de ses disciples et les conduire vers la vérité, renvoie à la fois aux dialogues de Platon et aux débats musicaux chers à E.T.A. Hoffmann (on pense au dialogue intitulé "Le poète et le compositeur » [Hoffmann 1963, p. 83 sq.]).

La deuxième partie, qui est en fait un second dialogue, met en scène des divinités de l'Olympe discutant de la valeur du compositeur Wagner et s'éloigne du sujet initial. Les discussions lors du premier dialogue sont centrées sur les premiers écrits (le premier volume des Écrits et poèmes réunis) - et la conversation laisse entendre que les personnages vont examiner systématiquement la totalité des dix volumes en cours de parution, mais la discussion se clôt en fait après l'examen de deux textes seulement : 1'« Esquisse autobiographique » et «De la musique allemande » ${ }^{14}$. Deux arguments principaux sont retenus contre Wagner : l'insincérité du compositeur, qui ne travaillerait qu'à sa propre gloire, et les contradictions de ses positions théoriques, qui apparaîtraient au grand jour à la lecture de l'essai intitulé " De la musique allemande ». Wagner fait en effet, dans ce texte de 1840, 1'apologie de la musique instrumentale pure (et reprend au passage une partie de l'argumentation développée par E.T.A. Hoffmann dans sa fameuse recension de la Cinquième symphonie de Beethoven [Hoffmann 1810]) avant de prendre fait et cause pour l'idée que la musique doit totalement se soumettre à la poésie pour avoir du sens. Glaukopides explique ce revirement par la volonté de trouver un succès plus facile dans le domaine de l'opéra et, pour mieux souligner l'absence d'éthique artistique de Wagner, il cite Robert Schumann : "Les lois de la morale sont également celles de $1^{\prime} \operatorname{art}^{15}$ "(Schumann 1850, p. 4).

\section{LA PREMIĖRE VERSION DU JUDAÏSME DANS LA MUSIQUE (1850)}

Curieusement, la première parution du tristement célèbre article sur « Le judaïsme dans la musique " (Wagner 1850) dans la Neue Zeitschrift für Musik n'a suscité que peu de réactions médiatiques en dehors de la revue elle-même, qui publie quelques réponses au pamphlet antisémite publié sous le pseudonyme K. Freigedank : celle d'Eduard Krüger, assez odieuse (Krüger 1850) ${ }^{16}$, celle d'Eduard Bernsdorf

\footnotetext{
14 «Über deutsches Musikwesen », dans SSD, t. 1, p. 149-166.

15 "Die Gesetze der Moral sind auch die der Kunst».

16 Krüger reprend à son compte l'argument de Wagner selon lequel les Juifs n'auraient aucune disposition pour la musique, mais tente de se montrer "magnanime " en suggérant que l'on fasse une exception pour Mendelssohn.
} 
qui souligne les inconséquences de l'argumentation de Wagner (Bernsdorf 1850), et celle de Brendel, qui essaie maladroitement de se dédouaner en invoquant la liberté de débattre (Brendel 1851a) ${ }^{17}$. De manière générale, l'article ne suscite pas les réactions que l'on serait en droit d'attendre. On trouve bien quelques remarques indignées dans la revue Europa. Chronik der gebildeten Welt, mais il s'agit d'une brève digression dans un article consacré à des récits juifs publiés à Vienne par Leopold Kompert : le journaliste s'insurge contre l'opprobre jeté sur la production artistique des Juifs à cause de l'article de la Neue Zeitschrift für Musik ${ }^{18}$ (Anonyme 1850a, p. 809). La seule réaction scandalisée et longuement argumentée que l'on puisse trouver est celle du compositeur et musicologue Johann Christian Lobe dans l'Illustrirte Zeitung de Leipzig :

Quelle est la substance de ce raisonnement de Freigedank? C'est : je hais les Juifs, je hais et je jalouse Mendelssohn et Meyerbeer, et je demande par conséquent qu'on anéantisse tous les Juifs. Celui qui n'est pas avec moi est un incapable, irréfléchi et insensible, il est lui aussi un Juif, et il doit être anéanti. - Est-ce que ce raisonnement peut sérieusement émaner d'un esprit sain et d'une âme humaine? Non (Lobe 1851 p. 56) $)^{19}$ !

Notons au passage que l'utilisation d'un pseudonyme pour publier des articles dans la presse était à l'époque une pratique courante et ne signifiait pas nécessairement que l'auteur ne voulait pas être identifié. Le nom de Wagner n'apparaît par conséquent pas dans les réponses mentionnées ci-dessus.

En revanche, la publication en mars 1869 de la version augmentée du texte sous le titre Éclaircissements sur le judä̈sme dans la musique (Aufklärungen über das Judenthum in der Musik; Wagner 1912b) provoque un déluge de réactions : un article paru dans 1'Allgemeine musikalische Zeitung en juillet de la même année dénombre au moins 25 recensions détaillées et huit publications séparées (il s'agit d'essais d'une longueur comprise entre 30 et 60 pages) parues en l'espace de trois mois (Anonyme 1869, p. 215). On peut s'interroger sur cette différence de traitement par rapport à l'article de 1850. Sans doute la célébrité désormais acquise par Wagner a-t-elle joué un rôle déterminant. À cela s'ajoute le fait que la question de l'émancipation des Juifs est entre-temps devenue un sujet dont on parle, notamment avec les discussions engagées

17 Dans son commentaire passablement embarrassé, Brendel essaie de faire dire à Wagner que, s'il inclut les Juifs dans le phénomène général de décadence artistique qui caractériserait son époque, il ne les tient pas pour les seuls responsables de ce phénomène. Dans une note qui accompagne l'article de Krüger, Brendel laisse toutefois entendre qu'il ne partage pas le point de vue de K. Freidedank, dont il déclare en même temps qu'il s'agit d'" un de nos plus importants compositeurs " ("von einem unserer bedeutendsten Tonsetzer»; Krüger 1850, p. 145).

18 "Herr Freigedank ging in seinem Galimathias so weit, den Juden, als unfähig aller wahren Leidenschaft, den Beruf zur Kunst streitig zu machen " ("Dans son galimatias, M. Freigedank est allé jusqu'à dénier aux Juifs, qui seraient incapables d'éprouver passion authentique, toute vocation artistique »).

19 "Was ist der Kern des ganzen Freigedank'schen Raisonnements? - dieser: Ich hasse die Juden; ich hasse und beneide Mendelssohn und Meyerbeer; ich rathe daher, alle Juden zu vernichten. Wer nicht mit mir ist, ist ein gedanken - und gefühlloser Schlendrianer. Kann ein solcher Raisonnement aus dem Geiste und dem Herzen eines vernünftigen, humanen Menschen unserer Zeit fließen? Nein! ». 
au parlement de Prusse à propos d'une loi sur l'égalité des droits des Juifs qui sera votée en juillet 1869 (Nipperdey 2013, t. 2/1, p. 401).

LES « ÉCRITS DE ZURICH » (1849-1851)

C'est surtout avec les « écrits de Zurich » sur la réforme de l'opéra que se crée un véritable espace médiatique autour des écrits du compositeur Richard Wagner.

Ces écrits, qui ont d'emblée fait apparaître de profonds clivages parmi leurs premiers lecteurs, ont logiquement fait l'objet de nombreux commentaires à la fois dans la presse généraliste et spécialisée et dans les ouvrages consacrés à la musique et à l'esthétique musicale qui fleurissaient en ce milieu de XIX ${ }^{e}$ siècle. Nous ne nous étendrons pas ici sur la réception des idées théoriques de Wagner dans les essais esthétiques et ouvrages de musicologie et d'histoire de la musique ; on constate toutefois, à la lecture des publications de Friedrich Theodor Vischer, d'Eduard Hanslick, d'Eduard Krüger, d'Adolf Bernhard Marx, d'August Wilhelm Ambros ou de Franz Brendel, que ce sont au moins autant les écrits réformateurs du compositeur que sa production musicale qui retiennent l'attention des esthéticiens et musicographes de l'ère post-hégélienne. Certains même - nous pensons au philosophe post-hégélien Friedrich Theodor Vischer ou au théoricien du formalisme Robert von Zimmermann - ne connaissaient manifestement Wagner qu'à travers ses écrits théoriques.

Nous entendons ici par " espace médiatique » l'émergence d'une sorte de forum de discussion virtuel où l'on peut identifier des problématiques récurrentes auxquelles un certain nombre d'articles s'intéresse en essayant de leur trouver des réponses à chaque fois différentes, et en prenant position les uns par rapport aux autres : ce phénomène contribue à créer un espace médiatique en ce sens où la communication ne circule pas uniquement verticalement, à savoir de l'artiste au lecteur par l'intermédiaire $\mathrm{du}$ journaliste, mais également horizontalement, entre les différentes publications. Plusieurs articles introduisent d'ailleurs leur sujet en justifiant leur prise de parole par la nécessité de répondre à telle ou telle autre publication dont, bien évidemment, ils ne partagent pas entièrement les analyses.

Prenons comme exemple les Écrits sur l'art de Richard Wagner (Richard Wagner's Schriften über Kunst), série de neuf articles publiés par Uhlig entre août 1850 et avril 1852, qui constituent l'une des premières publications journalistiques d'envergure ayant œuvré à la popularisation des textes de Wagner. L'auteur y présente en particulier L'art et la révolution, l'Ébauche d'organisation d'un théâtre national allemand pour le Royaume de Saxe ainsi qu'Opéra et drame (voir Uhlig 1851 et 1850-1852). D'emblée, les articles sont présentés comme une réponse argumentée aux commentaires publiés par un journal de Dresde dont le titre n'est pas précisé, et qui s'en serait pris à Wagner en lui reprochant son manque de propositions concrètes pour réformer l'opéra, et surtout sa remise en cause du christianisme. L'article auquel Uhlig fait allusion est sans doute une recension de L'art et la révolution parue dans le Dresdner Journal und Anzeiger. Il y est dit que Wagner semble vouloir « transformer les pierres des barricades de Dresde en 
pierres de construction pour la cathédrale de l'avenir ${ }^{20}$ »(Anonyme 1849a, p. 2152a), et le journaliste met en parallèle le projet du compositeur de faire revivre l'art antique et ses projets révolutionnaires. Il explique notamment que lorsque Wagner parle d'Apollon, il faut comprendre en réalité August Röckel, qui était un révolutionnaire proche de Mikhail Bakounine et a fait découvrir à Wagner les idées des premiers socialistes français. L'article du Dresdner Journal und Anzeiger est également commenté dans la revue Signale für die musikalische Welt, qui en cite de larges extraits (Anonyme 1849b, p. 349). Et il trouve également un écho plus diffus dans un papier consacré à L'art et la révolution par la Deutsche Allgemeine Zeitung de Leipzig et qui, développant un argumentaire analogue, critique à la fois la collusion entre le projet de réforme de l'opéra et la remise en cause de l'ordre public et étatique (Wagner chercherait à instrumentaliser la révolution à des fins carriéristes) et explique que le retour à l'Antiquité voulu par Wagner ne serait en réalité qu'un masque destiné à dissimuler une haine du christianisme qui, selon le journaliste, serait de nature "plus orientale qu'hellénique ${ }^{21} »$ (Anonyme 1850c, p. 117).

Le contenu des " écrits de Zurich ", consacrés pour l'essentiel à la réforme de l'opéra, est bien mieux connu que celui des écrits des années 1870 . On peut en résumer brièvement les lignes directrices : critique de la vie musicale de l'époque et de la conception traditionnelle de l'opéra, nécessité d'une réforme en profondeur de l'opéra et des institutions musicales, nécessité de remplacer les sujets historiques par le mythe, volonté de revenir aux fondamentaux du théâtre antique et de repenser la place du théâtre dans la société, nécessité de repenser le rapport entre texte et musique en partant de l'idée que les deux composantes doivent être mises au service du drame, développement de la théorie du Stabreim (vers allitératif), de la théorie du Leitmotiv (en réalité appelé Grundmotiv, «motif fondamental»), projet de fusion des arts, vision du monde marquée par la lecture de la philosophie de Feuerbach, sa critique du christianisme et sa métaphysique de l'amour.

Le terme de « réforme » revient souvent lorsqu'il est question des écrits de Wagner, c'est un terme qu'il emploie d'ailleurs lui-même régulièrement. Même sila comparaison est plus implicite qu'explicite, le jour sous lequel le cas Wagner est présenté dans la presse allemande suggère parfois un parallèle avec la Réforme de Luther ${ }^{22}$, dont les principales caractéristiques sont projetées sur le domaine de l'opéra : on trouve dans les deux cas une même critique virulente d'une situation indigne, le même rigorisme, la même volonté de revenir aux origines (dans un cas le christianisme, dans l'autre cas la tragédie antique), la même habileté à utiliser les moyens de communication modernes.

20 "Fast scheint es, als wolle Richard Wagner durch seine Broschüre „Die Kunst und die Revolution” auch die Dresdner Barricadensteine zu Bausteinen für den Dom der Zukunft verklären ».

21 "[Das Christenthum], dem Richard Wagner einen mehr orientalischen als hellenischen Haß geschworen hat ".

22 Voir : O. G. 1853, p. 138. La comparaison entre Wagner et Luther fera ultérieurement l'objet des sarcasmes d'Eduard Hanslick (Hanslick 1889, p. 65). Voir également "Sommerfahrten. Bayreuth ", Mährisches Tagblatt (Olmütz), 2 septembre 1882, p. 1. 
Si la totalité des articles consacrés aux écrits de Wagner publiés dans la presse allemande des années 1850 prennent position, de manière plus ou moins nuancée, pour ou contre ses théories réformatrices, la plupart des journalistes - qu'il s'agisse de la presse spécialisée ou de la presse généraliste - se comportent en pédagogues et se fixent comme objectif premier de rendre compte, de la façon la plus synthétique possible, du contenu et de l'argumentation développée dans les essais en question. C'est le cas notamment des articles de Uhlig ou bien de l'article, plus succinct, publié par Brendel en 1851 («Pour une évaluation des écrits de Richard Wagner»; Brendel 1851b) : tous deux expliquent qu'il faut s'en tenir aux grandes lignes des textes de Wagner et ne pas se focaliser sur des détails souvent discutables. Même si l'objectif de Uhlig est in fine de faire la promotion simultanée de la réforme wagnérienne et de ses convictions révolutionnaires (le terme communiste y est même prononcé), il affirme à plusieurs reprises qu'il veut avant tout proposer au lecteur une synthèse de L'art et la révolution et de L'ouvre d'art de l'avenir afin de dissiper les malentendus suscités par Wagner : " je vais dans un premier temps me contenter de suivre pas à pas les développements de Wagner ${ }^{23}$ "(Uhlig 1850-1852, 3 décembre, p. 241).

Même des articles extrêmement négatifs, comme les recensions d'Opéra et drame publiées début 1852 - quelques semaines après la publication de l'essai - dans l'hebdomadaire Die Grenzboten (Anonyme 1852a) et dans le quotidien NationalZeitung (Anonyme 1852b), s'évertuent à présenter les principales thèses exposées par le compositeur tout en respectant scrupuleusement l'ordre de l'ouvrage. Il s'agit en l'occurrence de démontrer que les idées de Wagner sont passablement excentriques et qu'il tente de plaquer de manière artificielle des principes totalement abstraits sur une réalité qu'il connaît mal.

On peut au final considérer comme acquis le fait que les conceptions esthétiques du compositeur ont été largement diffusées en Allemagne dès les années 1850 et que la majorité du public cultivé qui assistait à cette époque à une représentation de Lohengrin ou de Tannhäuser avait une idée relativement précise des principaux arguments exposés dans L'œuvre d'art de l'avenir ou Opéra et Drame - même si c'était parfois au prix de certaines distorsions, elles-mêmes à l'origine de diverses méprises.

\section{MALENTENDUS ET VISIONS RÉDUCTRICES}

Parmi les malentendus récurrents véhiculés par la presse autour des écrits de Wagner, on trouve en particulier le problème des rapport entre texte et musique : on lit dans de nombreux articles que le compositeur voudrait asservir la musique au texte, alors qu'en réalité il dit tout autre chose. On trouve, parmi les différents sujets de malentendus, le système des Leitmotive, auxquels on n'a pas encore donné ce nom, mais dont on dit que son application systématique constitue un carcan contraire à l'invention musicale; ou encore de la question de la « musique absolue ${ }^{24}$ », qui fait

23 "[Ich werde] mich vorerst begnügen [...], den Entwicklungen W's Schritt vor [sic] Schritt zu folgen ».

24 L'analyse la plus complète et la plus pertinente de la notion de musique absolue reste l'ouvrage de Carl Dahlhaus, Die Idee der absoluten Musik (Dahlhaus 1994). On pourra également se référer à nos propres remarques sur le sujet dans Candoni 2012, p. 36-40. 
déjà l'objet de débats dans lesquels on confond le plus souvent "musique absolue " et " musique instrumentale » ou bien " mélodie absolue » et " inventivité musicale » - alors que Wagner comprend en réalité cette notion de " musique absolue » dans une perspective essentiellement feuerbachienne : l'absolu est pour lui une abstraction néfaste qui ignore les réalités humaines et historiques. C'est ainsi qu'on peut lire dans une recension de Tannhäuser publiée dans un journal du soir viennois : "Wagner dédaigne la mélodie absolue, et la musique absolue en général ? La mélodie absolue et la musique absolue le lui rendent bien ! Disons-le franchement : sa force créatrice musicale et son véritable réservoir d'idées se réduisent au strict minimum ${ }^{25}$ " (C. D. 1857 , p. 882 ).

À côté de ces malentendus, on relève de flagrantes simplifications, notamment lorsque les idées de Wagner sont réduites à des slogans qui, certes, participent à la diffusion de la "marque Wagner ", mais qui surtout prêtent le flanc à la caricature ou à diverses moqueries. On passe alors d'un espace médiatique de discussion à un espace médiatique purement publicitaire, qui évacue le débat au profit de préjugés. C'est évidemment le cas du terme de «musique de l'avenir », qui n'est pas de Wagner, mais qui est une extrapolation terminologique réalisée à partir du concept d' « œuvre d'art de l'avenir ». Le compositeur s'est d'ailleurs indigné, et sans doute était-il sincère, de l'utilisation abusive qui était faite du terme de « musique de l'avenir » dans la presse des années 1850 et 1860 . Il publie en 1860 un essai intitulé «Zukunftsmusik », version allemande de la "Lettre sur la musique » rédigée à l'intention du public français, dans lequel il réfute énergiquement ce qualificatif (l'utilisation des guillemets signale que l'auteur refuse de reprendre l'expression à son compte). On dit souvent, sur la foi de Wagner, que le terme de " musique de l'avenir » serait dû initialement au musicographe Ludwig Bischoff, qui l'aurait introduit en 1859 dans la Rheinische Musikzeitung (Wagner 1912b, p. 248) - en réalité, l'expression était très répandue dès le début des années 1850, c'est-à-dire à partir de la publication de L'œuvre d'art de l'avenir. Elle avait certes pour fonction de résumer en un slogan parlant le projet complexe de la réforme wagnérienne, mais elle était également prétexte à d'innombrables remarques ironiques ou désobligeantes. C'était le cas en particulier dans la presse viennoise, notamment dans la revue Der Humorist, extrêmement sarcastique envers Wagner (ex. : Anonyme 1857a) - et c'est en partie pour endiguer le flot de moqueries sur la "musique de l'avenir » et pour relancer une véritable discussion que Brendel a lancé le concept de Neudeutsche Schule en 1859 (voir Kleinertz 2006, p. 25).

En Allemagne comme en France, Wagner est donc devenu célèbre au moins autant par ses écrits esthétiques et politiques que par ses drames musicaux. Un article du rédacteur en chef de la Neue Berliner Musikzeitung, Carl Kossmaly, affirme même en 1855 : «par un pur hasard, le public a découvert ses textes théoriques avant de

25 "Wagner verachtet die absolute Melodie, die absolute Musik überhaupt? Die absolute Melodie, die absolute Musik erwiedern Gleiches mit Gleichem. Es muß offen ausgesprochen werden, daß sich Wagner's musikalische Gestaltungskraft, sein eigentlicher Ideenfond auf ein Minimum reduziren ". 
découvrir Tannhäuser et Lohengrin ${ }^{26} »$ (Kossmaly 1855, p. 258). L'auteur explique que ce qu'il appelle le « mouvement wagnérien » ("die Wagner-Bewegung ») s'est construit à partir de deux événements fondateurs : la création de Lohengrin (août 1850) et la parution des essais L'ouvre d'art de l'avenir (1849) et Opéra et drame (1851). Dire que les idées de Wagner ont été connues avant ses opéras est sans doute légèrement exagéré (même si ce n'est pas entièrement faux) : la première vague importante d'articles consacrés à Wagner dans la presse allemande remonte à la création de Rienzi (1842), à une époque où le compositeur n'avait pas encore publié ses grands textes sur l'art, la révolution et la réforme de l'opéra.

Évaluer l'importance des écrits de Wagner dans la réception de son œuvre ne se limite toutefois pas à une question de chronologie et d'antériorité : le XIX siècle est, par excellence, le siècle où l'intention de l'auteur fait autorité sur la réception de son œuvre (c'est d'ailleurs une notion centrale d'Opéra et drame : l'œuvre d'art est, selon Wagner, la « réalisation d'une intention poétique ${ }^{27}$ "; Wagner 1912-1914, t. 4, p. 118), et l'idée de laisser à l'auteur le soin de commenter son œuvre ne suscitait à l'époque pas les mêmes réserves qu'aujourd'hui. Attention : cela ne signifie pas qu'on ne puisse pas mettre en avant les contradictions ou incohérences entre l'œuvre artistique et les écrits théoriques. C'est le cas par exemple d'un article publié dans la Illustrirte Zeitung en février 1865, qui souligne l'écart entre les prétentions intellectuelles de la théorie et une œuvre musicale qui défie les lois de la raison (P. L. 1865, p. 132). Seulement, le constat d'une inadéquation entre l'œuvre et la théorie amène le plus souvent à la conclusion que l'œuvre comporte de nombreuses faiblesses - alors qu'il serait parfois plus pertinent d'évaluer l'œuvre en question indépendamment des textes théoriques, dans la mesure où ces derniers peuvent faire écran entre l'œuvre et son commentateur. On peut en tout état de cause constater, dans la presse allemande des années 1850, que non seulement un espace important est consacré aux écrits théoriques de Wagner, mais que les critiques de ses opéras sont fréquemment rédigées à la lumière des « écrits de Zurich », ou de l'opinion qu'on s'en est fait au préalable.

Prenons l'exemple d'un compte rendu de la première représentation de Tannhäuser au Thalia-Theater de Vienne en août 1857 : la recension, publiée dans le quotidien Der Humorist, consiste pour l'essentiel en une suite de remarques ironiques sur la " musique de l'avenir" (avec de nombreuses allusions qui échappent au lecteur d'aujourd'hui) et sur la prétention de Wagner à faire table rase de l'histoire de la musique pour se placer lui-même sur le trône de l'art. Ce n'est que dans les toutes dernières lignes de l'article qu'il est enfin question de l'opéra Tannhäuser : l'auteur anonyme regrette que le compositeur reste fidèle à la doctrine selon laquelle l'individuel doit être sacrifié au collectif. Il s'agit à l'évidence d'une lecture incorrecte de L'ouvre d'art de l'avenir, l'essai étant manifestement perçu comme un texte de propagande communiste. L'auteur du compte rendu écrit : " cet axiome est la raison pour

26 "Ganz zufällig habe das Publikum die genannten Schriften früher zur Hand bekommen, als es die Opern Tannhäuser und Lohengrin gehört». L'auteur de l'article reprend ici le point de vue de Joachim Raff.

27 "Verwirklichung der dichterischen Absicht». 
laquelle le texte de Tannhäuser, c'est-à-dire le véritable drame, est incapable de faire progresser 1'action ${ }^{28}$ " (Anonyme 1857b, p. 929); et il ajoute : " les situations sont de ce fait tellement étirées que la musique est incapable de les saisir, la partie musicale se relâche et son efficacité faiblit ${ }^{29}$ " (ibid.). On comprend mal le raisonnement qui permet d'établir ce rapport entre théorie et musique, mais les faiblesses de la musique sont à l'évidence imputées à la rigidité de la théorie, Wagner étant accusé « de vouloir faire entrer de force l'expression musicale dans un système » et de chercher en fin de compte à «transformer l'art en une science ${ }^{30}$ " (ibid.). L'année suivante, on constate le même type de réaction lors de la création viennoise de Lohengrin : la recension parue dans le quotidien viennois Morgen-Post est pour moitié un rappel des théories de Wagner, le jugement émis sur l'œuvre servant ensuite essentiellement à démontrer l'absurdité de la réforme wagnérienne et a contrario, la nécessité de s'en tenir aux formes traditionnelles de l'opéra (Anonyme 1858, p. 2).

Notre second exemple est emprunté à un volumineux article publié par Kossmaly dans la Neue Berliner Musik-Zeitung et consacré à ce qu'on appelle déjà " la question Wagner » (" die Wagnerfrage »; Kossmaly 1855-1856). Il s'agit d'une longue discussion autour d'un ouvrage consacré à Wagner par le compositeur et musicologue Joachim Raff, dont le journaliste partage les principales positions malgré des désaccords ponctuels. L'article propose une vision des choses beaucoup plus nuancée que celle du Morgen-Post, mais il prouve, une fois encore, que l'œuvre musicale est jugée à travers le prisme des écrits théoriques du compositeur. Kossmaly explique (en s'appuyant sur l'argumentation de Raff) que le projet artistique de Wagner repose sur la combinaison de la spéculation, qui lui permet de dépasser son époque, et de l'imagination, qui lui permet de concrétiser le résultat de cette spéculation sans toutefois éviter le risque de tomber dans le dilettantisme. Tout au long de cet article, qui n'est ni résolument hostile, ni complètement favorable à Wagner, Kossmaly affirme vouloir faire la part des choses entre la théorie et la pratique artistique, et insiste sur le fait que la conception de Lohengrin, dont il propose une minutieuse analyse musicale, est antérieure à la rédaction des écrits de Zurich, dans lesquels on ne saurait voir autre chose qu'une déclaration d'intention dans la perspective des opéras à venir. Et pourtant, on découvre bientôt, en suivant les méandres de son raisonnement, qu'il explique les prétendues faiblesses de la musique de Lohengrin par la volonté du compositeur de transposer de façon inflexible et systématique ses principes théoriques dans sa pratique compositionnelle : la mise en œuvre de la théorie des «motifs fondamentaux " exposée dans Opéra et drame (Kossmaly parle de "Motivierungstheorie ») ne permet pas, selon lui, de

28 "Dieser Grundsatz ist die Ursache, daß im Texte des „,Tannhäuser" das eigentliche dramatische Leben, der wahre Fortgang einer Handlung nicht merkbar wird".

29 "Die Gedehntheit der Situationen ist auch Schuld, daß die Musik sich nicht festzuhalten vermag, die Tonlichkeit erschlafft und mit ihr schwächt sich auch die Wirksamkeit $a b$ ».

30 "Würde Wagner einen ergiebigen Quell der Melodie in sich verspüren, wäre er wahrscheinlich nicht auf die Idee verfallen, auf einem neuen Wege zu dem Höhepunkte der Bestimmtheit des musikalischen Ausdruckes zu gelangen, diesen in ein neues System zu zwängen, welches, wenn es auch die Durchführbarkeit in sich bärge, die Musik aus einer Kunst zu einer Wissenschaft machen würde». 
soutenir l'attention de l'auditeur pendant toute la durée de l'opéra ; le « communisme » de Wagner, qui se traduit au premier acte de Lohengrin par un communisme musical entre Elsa et le chœur (en ce sens où ils doivent partager un même thème musical) empêcherait que les deux parties soient clairement caractérisées et différenciées ; ce même communisme musical pousserait Wagner à privilégier l'homophonie par rapport à la polyphonie (dans les chœurs notamment), ce qui ne serait au fond qu'un expédient visant à faire oublier sa maîtrise insuffisante de l'écriture polyphonique (c'est « le travail d'un maître certes intelligent et bien intentionné, mais qui n'a pas reçu une formation suffisante ${ }^{31}$ "; ibid., 5 décembre, p. 386) ; le système des "motifs fondamentaux ", appliqué de façon méthodique, constituerait un obstacle à la fluidité et à l'élan du discours musical, d'autant plus que, conformément à la théorie exposée dans Opéra et drame, le soin de caractériser les personnages incomberait à l'orchestre au détriment des chanteurs, devenus inexpressifs.

$* * *$

Le moment où Wagner entre au cœur des débats, au début des années 1850, coïncide avec la publication de ses écrits réformateurs, dont l'impact sur l'opinion publique ne saurait être sous-estimé. Leur caractère radical et provocateur a largement contribué à faire naître une discussion animée autour du phénomène Wagner, même s'il faut bien reconnaître que les écrits du compositeur, souvent déformés ou incompris, ont pu constituer un obstacle à une réception nuancée et objective de ses drames musicaux autant qu'ils ont aidé le public à comprendre la cohérence du projet d' " ouvre d'art de l'avenir ». L'étude de la réception des écrits du compositeur dans la presse allemande de son époque, qui est ici seulement esquissée et devra être poursuivie sur une période plus longue ${ }^{32}$, démontre en tout état de cause que son œuvre a été perçue par ses contemporains comme un phénomène global, dans lequel théorie esthétique et production artistique sont indissociables, initiant ainsi un type d'exégèse dont se réclamera la critique allemande jusqu'au XXI siècle. On s'étonnera par ailleurs de la faible présence des enjeux nationalistes dans les débats suscités par Wagner à l'époque prise ici en considération. Il ne faut toutefois pas oublier que les écrits rédigés dans les années 1840 et 1850 sont essentiellement pensés dans une perspective révolutionnaire - et souvent même universaliste - et que la question nationale n'apparaîtra avec insistance qu'à partir des textes des années 1860 (Qu'est-ce qui est allemand?, 1865 ; Wagner 1912c) et 1870 (Beethoven, 1870 ; Wagner 1912d). Notons néanmoins que l'animosité quasiment systématique de la presse viennoise envers le musicien saxon ${ }^{33}$

31 "Das Erzeugnis eines zwar geistreichen und wohl intentionirten, aber nicht genügend ausgebildeten Musikers".

32 Pour une étude générale, mais n'accordant qu'une place très secondaire aux écrits du compositeur, sur la réception du "phénomène Wagner » dans le monde germanique de la seconde moitié du XIX ${ }^{e}$ siècle, voir Müller 2013, p. 25-90.

33 Les origines saxonnes de Wagner ont certainement joué un rôle dans son aversion pour la Prusse (tenue notamment pour responsable de la répression du soulèvement de Dresde en 1849), et dans ses relations compliquées avec Vienne, la patrie du formalisme musical. 
peut être replacée dans le contexte de la rivalité grandissante entre l'Allemagne protestante, dont la Prusse était le centre de gravité, et une Autriche catholique traditionnellement hostile à la notion d'œuvre d'art total (voir Hofmann 1989, p. 84-85) même si, loin s'en faut, Wagner n'a jamais été un partisan de la politique prussienne.

\section{BiBLIOGRAPHIE}

Anonyme (1849a), [sans titre], Dresdner Journal und Anzeiger, 30 septembre, p. 2152a.

Anonyme (1849b), [sans titre], Signale für die musikalische Welt (Leipzig), n 44 , octobre, p. 349.

Anonyme (1850a), «Deutsches Judenthum und christliche Kritik», Europa. Chronik der gebildeten Welt (Leipzig), 21 décembre, p. 809-813.

Anonyme (1850b), " Richard Wagner. Das Kunstwerk der Zukunft », Beilage zum Morgenblatte der Wiener Zeitung, 30 avril, [n. p.].

Anonyme (1850c), [sans titre], Deutsche Allgemeine Zeitung (Leipzig), 15 janvier, p. 117.

Anonyme (1852a), «Das Verhältniß der Oper zum Drama », Die Grenzboten (Leipzig), 9 janvier, p. 81 94.

Anonyme (1852b), «Oper und Drama. Von Richard Wagner », National-Zeitung (Berlin), 6 avril, p. AaBc ; 10 juin, p. Aa-Cc.

Anonyme (1857a), «Die wöchentliche Rückschau von Jocosus Krebsimanus », Der Humorist (Vienne), 25 février, p. 196-197.

Anonyme (1857b), «Thalia-Theater. Zum 1. Male: „Tannhäuser und der Sängerkrieg auf der Wartburg" ", Der Humorist (Vienne), 30 août, p. 928-929.

Anonyme (1858), « Richard Wagner's Lohengrin », Morgen-Post (Vienne), 20 août, p. 2.

Anonyme (1869), «Die musikalische Judenfrage », Allgemeine musikalische Zeitung (Leipzig), 7 juillet, p. 215.

Bagge, Selmar (1861), «Tristan und Isolde von Richard Wagner », Deutsche Musik-Zeitung (Vienne), 23 février, p. 57-61.

Bermbach, Udo (2013), Mythos Wagner, Berlin, Rowohlt.

Bernsdorf, Eduard (1850), " K. Freigedank und das Judenthum in der Musik », Neue Zeitschrift für Musik [=NZfM] (Leipzig), 15 octobre, p. 165-168.

Brendel, Franz (1851a), « Das Judenthum in der Musik », NZfM, 4 juillet, p. 4-6.

Brendel, Franz (1851b), «Zur Beurtheilung der Schriften Richard Wagner's », NZfM, 14 septembre, p. 209-211; 28 novembre, p. 230-234.

Candoni, Jean-François (2012), Penser la musique au siècle du romantisme. Discours esthétiques dans l'Allemagne et l'Autriche du XIX' siècle, Paris, PUPS.

C. D. (1857), «Richard Wagner's Tannhäuser », Abendblatt der Oesterreichisch-Kaiserlichen Wiener Zeitung, 28 septembre, p. 881-882.

Dahlhaus, Carl ([1978]1994), Die Idee der absoluten Musik, Kassel, Bärenreiter.

Döhring, Sieghart, et Sabine Henze-Döhring (2014), Giacomo Meyerbeer. Der Meister der Grand Opéra, München, C. H. Beck.

Fischer, Jens Malte (2000), Richard Wagners „Das Judentum in der Musik“, Frankfurt am Main, C. H. Beck.

Garratt, James (2010), Music, Culture and Social Reform in the Age of Wagner, Cambridge, Cambridge University Press. 
Hanslick, Eduard (1889), «Was denken Sie von Wagner? », dans Eduard Hanslick, Musikalisches und Literarisches. Kritiken und Schilderungen, Berlin, Allgemeiner Verlag für Deutsche Literatur, p. 5666.

Hoffmann, E.T.A. (1810), "Recension. Sinfonie... par Louis van Beethoven », Allgemeine musikalische Zeitung (Leipzig), 4 juillet, col. 630-642 ; 11 juillet, col. 652-659.

Hoffmann, E.T.A. (1963), Die Serapions-Brüder, München, Winkler Verlag.

Hofmann, Werner (1989), "Was ist deutsch an der deutschen Kunst? », dans Werner Hofmann, Anhaltspunkte Studien zur Kunst und Kunsttheorie, Frankfurt am Main, Fischer, p. 79-88.

Kalischer, Alfred (1872a), "Kritischer Dialog über Richard Wagner's „Gesammelte Schriften und Dichtungen" ", Neue Berliner Musikzeitung, 3 janvier, p. 2-5 ; 10 janvier, p. 11-14 ; 17 janvier, p. 1719 ; 24 janvier, p. 25-27; 31 janvier, p. 33-35; 7 février, p. 41-43 ; 14 février, p. 49-51.

Kalischer, Alfred (1872b), "Zweiter Dialog über Richard Wagner's „Schriften und Dichtungen“", Neue Berliner Musikzeitung, 2 octobre, p. 314-315 ; 16 octobre, p. 329-331 ; 30 octobre, p. 345-347.

Kirchmeyer, Helmut (1967-1985), Das zeitgenössische Wagnerbild, 5 tomes, Regensburg, Bosse.

Kleinertz, Rainer (2006), "Zum Begriff „Neudeutsche Schule“”, dans Detlef Altenburg (dir.), Liszt und die Neudeutsche Schule, Laaber, Laaber Verlag, p. 23-31.

Kossmaly, Carl (1855-1856), « Die Wagnerfrage von Joachim Raff. Kritisch beleuchtet von C. Kossmaly ", Neue Berliner Musikzeitung, 15 août, p. 257-300 ; 22 août, p. 265-268 ; 29 août, p. 273-276 ; 5 septembre, p. 281-284 ; 12 septembre, p. 289-292 ; 19 septembre, p. 298-300 ; 24 septembre, p. 305-308 ; 28 novembre, p. 377-378 ; 5 décembre, p. 385-386 ; 12 décembre, p. 393394 ; 19 décembre, p. 401-402 ; 9 janvier, p. 9-10 ; 23 janvier, p. 25-26 ; 21 mai, p. 161-163 ; 29 mai, p. $169-171 ; 4$ juin, p. 177-179; 11 juin, p. 185-187.

Krüger, Eduard (1850), « Judenthümliches », NZfM, $1^{\text {er }}$ octobre, p. 145-147.

Lobe, Johann Christian (1851), «Das Judenthum in der Musik », Illustrirte Zeitung (Leipzig), 25 janvier, p. 54-56.

Müller, Sven Oliver (2013), Richard Wagner und die Deutschen. Eine Geschichte von Hass und Hingabe, München, C. H. Beck.

Nattiez, Jean-Jacques (2015), Wagner antisémite. Un problème historique, sémiologique et esthétique, Paris, Christian Bourgois.

Nipperdey, Thomas (2013), Deutsche Geschichte 1800-1866, 3 tomes, München, C. H. Beck.

O. G. (1853), "Reflectionen über die Oper „der Tannhäuser" von Richard Wagner », Neue Berliner Musikzeitung, 28 mai, p. 137-140.

P. L. (1865), « Richard Wagner », Illustrirte Zeitung (Leipzig), 25 février, p. 131-133.

Picard, Timothée (dir.) (2010), Dictionnaire encyclopédique Wagner, Arles, Actes Sud.

Schumann, Robert (1850), « Musikalische Haus- und Lebensregeln », NZfM, 3 mai, Beilage zu Nr. 36, p. $1-4$.

Uhlig, Theodor (1851), «Aus Richard Wagner's „Entwurf zur Organisation eines deutschen NationalTheaters für das Königreich Sachsen" ", NZfM, 3 janvier, p. 2-3 ; 7 février, p. 50-52 ; 7 mars, p. 9799.

Uhlig, Theodor (1850-1852), « Richard Wagner's Schriften über Kunst », NZfM, $1850: 20$ août, p. 7680 ; 3 décembre, p. 241-245; 13 décembre, p. 261-262 ; 1851 ; 9 janvier, p. 13-16 ; 16 janvier, p. $25-$ $29 ; 17$ janvier, p. $21-24 ; 24$ janvier, p. 33-36; $1852: 1^{\text {er }}$ janvier, p. $4-6 ; 9$ janvier, p. 13-16 ; 16 janvier, p. $25-29 ; 16$ avril, p. 181-184.

Wagner, Richard (1850), "Das Judenthum in der Musik », NZfM, 3 septembre, p. 101-107 ; NZfM, 6 septembre, p. 109-112, https://dicteco.huma-num.fr/fr/article/29236, consulté le 17 septembre 2019.

Wagner, Richard (1912a), «Über musikalische Kritik, Brief an den Herausgeber der „Neuen Zeitschrift für Musik"», dans $S S D$, t. 5, p. 53-65. 
Wagner, Richard (1912b), Aufklärungen über das Judenthum in der Musik, dans SSD, t. 8, p. 238-261.

Wagner, Richard (1912c), Was ist deutsch?, SSD, t. 10, p. 36-54.

Wagner, Richard (1912d), Beethoven, SSD, t. 9, p. 61-127.

Wagner, Richard (1912e), Über deutsches Musikwesen, SSD, t. 1, p.149-166.

Wagner, Richard (1871-1883), Gesammelte Schriften und Dichtungen, 10 tomes, Leipzig, E.W. Fritzsch Verlag.

Wagner, Richard (1912-1914), Sämtliche Schriften und Dichtungen, 6 $6^{\mathrm{e}}$ édition, 16 tomes, Leipzig, Breitkopf \& Härtel/C. F. W. Siegel (R. Linnemann) [= SSD], https://dicteco.huma-num.fr/fr/ anthology/29135, consulté le 17 septembre 2019.

Zimmermann, Reiner (1998), Giacomo Meyerbeer. Eine Biographie nach Dokumenten, Berlin, Parthas Verlag. 